\title{
Laser-assisted electron scattering on a nano-sphere
}

\author{
Sándor Varróa, ${ }^{\mathrm{a},}$, Lóránt Zs. Szabóc ${ }^{\mathrm{c}}$, Attila Czirják ${ }^{\mathrm{b}, \mathrm{c}}$ \\ ${ }^{a}$ Wigner Research Center of the Hungarian Academy of Sciences, \\ Konkoly Thege Miklós út 29 - 33, Budapest 1121, Hungary \\ ${ }^{b}$ ELI-HU Nonprofit Kft., Dugonics tér 13, 6720 Szeged, Hungary \\ ${ }^{c}$ Department of Theoretical Physics, University of Szeged, Tisza Lajos körút 84, 6720 Szeged, Hungary
}

\begin{abstract}
We investigate the scattering of electrons on a hard sphere in the presence of a laser field of arbitrary intensity. We use spherical Gordon-Volkov states, and we present a novel method for the computation of a key quantity in this theory. We compute and analyse some additional results regarding the total differential scattering cross sections in the case of the weak field limit.
\end{abstract}

Keywords: Laser-assisted scattering, electron scattering on nano-particles, Gordon-Volkov states, multiphoton processes

PACS: 03.65.Nk, 32.80.Wr, 34.80.Qb, 42.50.Hz

\section{Introduction}

Laser-assisted electron scattering has been widely studied in the past, primarily in the context of multiphoton Bremsstrahlung and plasma heating [1]. Recently the application of this process has received a growing importance in various branches of research aiming, for instance, the generation of ultrashort (even attosecond) electron pulses [2, 3, 4], four-dimensional imaging and ultrafast electron microscopy $[5,6]$, or photon-induced near field electron microscopy $[7,8,9]$. The theoretical description of laser-assisted scattering processes of charged particles relies on the non-perturbative treatment of the interaction with the laser field [10, 11, 12], which is usually based on the Volkov states being modified de Broglie plane waves. The exact analytic treatment of this problem has already been carried out in an earlier study by one of the authors [10], and several closed-form results have been derived there.

In this contribution, we study a simple model for electron scattering on a nano-particle in the presence of a laser field. After summarizing the theoretical framework of [10], we present a novel method for the accurate and effective computation of a key quantity $P^{m}$ in the matching equations that determine the quantum state of the scattered electrons. We compute and analyse the Varró) electron wave function and the total differential scattering cross sections in the weak field limit, and draw some conclusions.

\section{Model and solution}

We consider electron scattering on a nano-particle in the presence of a low-frequency laser field, modelled as a plane wave with linear polarization in the $z$ direction, see Figure 1. The electrons are considered independent and they are described by the Schrödinger equation, their interaction with the laser field is taken into account by the usual minimal coupling, and we choose the Coulomb gauge. The long wavelength of the laser field justifies the use of the dipole approximation, thus the vector potential is

$$
\mathbf{A}=\left(0,0, A_{0} \cos \omega t\right)
$$

The incident electrons of charge $-e$ and mass $M$ propagate in an arbitrary direction defined by the polar angles $\Theta_{0}$ and $\varphi_{0}$. By means of a well-known unitary transformation, we can eliminate the interaction term $e^{2} A^{2} / 2 M c^{2}$ and hence the relevant part of the wave function of our scattering problem obeys the following Schrödinger equation:

$$
\left[\frac{\hat{\mathbf{p}}^{2}}{2 M}+\frac{e}{M c} \mathbf{A} \cdot \hat{\mathbf{p}}\right] \Psi=i \hbar \frac{\partial}{\partial t} \Psi,
$$




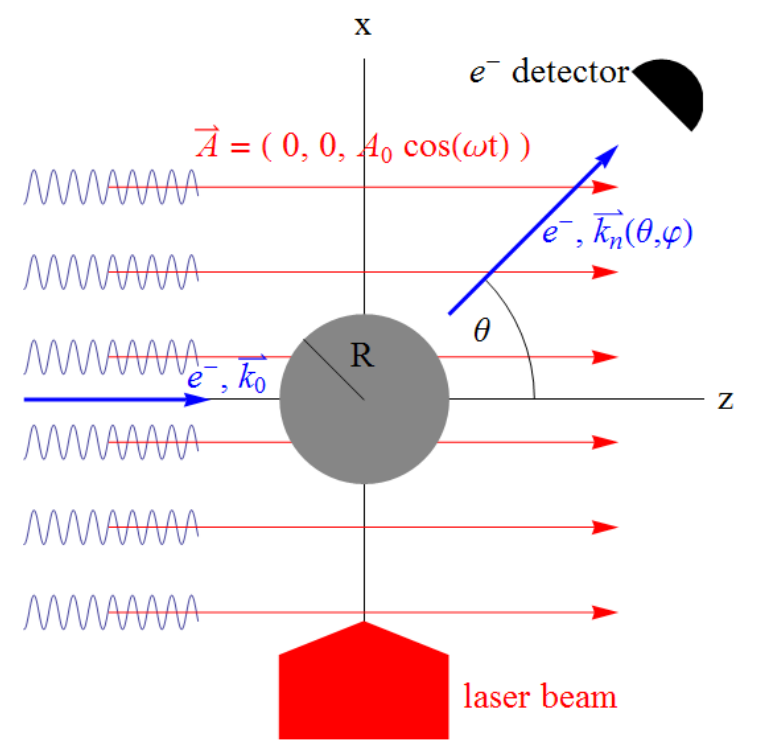

Figure 1: Geometry of the model for electron scattering on a nanoparticle in the presence of a linearly polarized laser field, see the text for details.

where $\hat{\mathbf{p}}=-i \hbar \nabla$. With the help of the KramersHenneberger transformation

$$
\Psi=\exp \left[-a \sin \omega t \nabla_{z}\right] \Phi(x, y, z ; t),
$$

the Schrödinger equation (2) is transformed into the free-particle Schrödinger equation:

$$
-\frac{\hbar^{2}}{2 M} \nabla^{2} \Phi=i \hbar \frac{\partial \Phi}{\partial t}
$$

The space-translation of the solution $\Phi$ of (4) as

$$
\Psi=\Phi(x, y, z-a \sin \omega t ; t)
$$

yields the solution $\Psi$ of (2) in the laboratory frame (see Fig. 1). Here $a=e A_{0} / M \omega c=\mu \lambda$, where $\mu=e A_{0} / M c^{2}$ is the intensity parameter.

In order to facilitate analytic treatment, we assume that the scattering target is a hard sphere of radius $R$ on which the potential $V=\infty$ and outside of which $V=0$. Thus, we impose the following boundary condition on our space-translated solution (5):

$$
\left.\Phi(x, y, z-a \sin \omega t ; t)\right|_{r=R}=0,
$$

which must hold for all times $t$ and all polar angles $\Theta$ and $\varphi$.

\subsection{Solution by expansion on spherical Gordon-Volkov states}

In order to satisfy the condition (6), it is convenient to introduce spherical polar coordinates $(r, \Theta, \varphi)$ taking as usual the $z$-axis as the polar axis. Writing down the Schrödinger equation (2) in these coordinates, the term representing the interaction with the radiation field is independent of the azimuth $\varphi$ as is the boundary condition (6). However, if the incoming electron wave will be chosen to be dependent on $\varphi$, then the total scattering wave function must be $\varphi$ dependent as well, which means there is no cylindrical symmetry with respect to the $z$-axis. If we write $\mathbf{r} \equiv r(\sin \Theta \cos \varphi, \sin \Theta \sin \varphi, \cos \Theta)$ and similarly represent the wave vector of the ingoing free electron by $\mathbf{k}_{0} \equiv k_{0}\left(\sin \Theta_{0} \cos \varphi_{0}, \sin \Theta_{0} \sin \varphi_{0}, \cos \Theta_{0}\right)$ then we find $\mathbf{k}_{0} \cdot \mathbf{r}=k_{0} r\left(\sin \Theta_{0} \sin \Theta \cos \varphi-\varphi_{0}+\cos \Theta \cos \Theta_{0}\right)$ and therefore the cylindrical symmetry can only be obtained if we choose $\Theta_{0}=0$, and this corresponds to an electron beam coming in along the vector of linear polarization of the laser field. This is also a configuration for which a maximum of interaction between the electron and radiation field can be expected.

In view of these considerations, we attempt to solve (4) by the ansatz

$$
\begin{aligned}
& \Phi=\exp \left[i\left(\mathbf{k}_{0} \cdot \mathbf{r}-\omega_{0} t\right)\right]+\sum_{l=0}^{\infty} \sum_{m=-l}^{l} \sum_{n=-\infty}^{\infty} h_{l}^{(1)}\left(k_{n} r\right) \\
& P_{l}^{m}(\cos \Theta) \exp (i m \varphi) A(n, l, m) \exp \left[-i\left(\omega_{0}+n \omega\right) t\right],
\end{aligned}
$$

where $E_{0}=\hbar \omega_{0}$ is the energy of the scattered electrons in the absence of the laser field and $h_{l}^{(1)}\left(k_{n} r\right) Y_{l}^{m}(\Theta, \varphi)=$ $f_{n, l, m}(r, \Theta, \varphi)$ are outgoing spherical waves satisfying the Helmholtz equation

$$
\left\{\nabla^{2}(r, \Theta, \varphi)+k_{n}^{2}\right\} f_{n, l, m}=0 .
$$

The $h_{l}^{(1)}\left(k_{n} r\right)$ are spherical Hankel functions of the first kind and the $Y_{l}^{m}(\Theta, \varphi)$ are ordinary spherical harmonics. In the laser field, the wave numbers $k_{n}$ of the scattered electrons are given by

$$
k_{n}=\frac{\sqrt{2 M\left(E_{0}+n \hbar \omega\right)}}{\hbar}=k_{0} \sqrt{1+n \frac{\omega}{\omega_{0}}},
$$

which can be real or purely imaginary, depending on the value of the integer $n$ for a given $\omega / \omega_{0}$. If the $k_{n}$ are purely imaginary, the spherical Hankel functions represent exponential decay of the partial waves. This means that the laser field can induce evanescent partial electron waves bound to the surface of the sphere. In (7) the 
coefficients $A(n, l, m)$ are so far unknown and have to be determined by means of the boundary condition (6).

Because of (5) and (7) the total wave function $\Psi$ can be written in the space-translated form

$$
\begin{aligned}
\Psi= & \exp \left\{i\left[\mathbf{k}_{0} \cdot \mathbf{r}-k_{0} \cos \Theta_{0} a \sin \omega t\right]\right\} \\
& +\sum_{l=0}^{\infty} \sum_{m=-l}^{l} \sum_{n=-\infty}^{\infty} A(n, l, m) h_{l}^{(1)}\left[k_{n} r(t)\right] \\
& \cdot P_{l}^{m}[\cos \Theta(t)] \exp (i m \varphi) \exp \left[-i\left(\omega_{0}+n \omega\right) t\right],
\end{aligned}
$$

where $r(t)$ and $\Theta(t)$ are the space-shifted polar coordinates. According to (10), the wave function is a superposition of an incoming plane Gordon-Volkov state $[13,14]$ and outgoing spherical Gordon-Volkov states with energies $E_{0}+n \hbar \omega$, corresponding to stimulated photon emission and absorption. In (10), the explicit expressions for $r(t)$ and $\Theta(t)$ are given by

$$
\begin{gathered}
r(t)=\sqrt{r^{2}-2 r \alpha(t) \cos \Theta+\alpha(t)^{2}} \\
\cos \Theta(t)=\frac{z-\alpha(t)}{r(t)}=\frac{r \cos \Theta-\alpha(t)}{\sqrt{r^{2}-2 r \alpha(t) \cos \Theta+\alpha(t)^{2}}}
\end{gathered}
$$

where

$$
\alpha(t)=a \sin \omega t, \quad a=\frac{e A_{0}}{M \omega c}=\mu \lambda .
$$

In order to be able to evaluate the coefficients $A(n, l, m)$ from the boundary condition (6), we first have to determine the explicit form of the spherical Gordon-Volkov states $f_{n, l, m}(r(t), \Theta(t), \varphi)=h_{l}^{(1)}\left(k_{n} r(t)\right) Y_{l}^{m}(\Theta(t), \varphi)$ in terms of the ordinary spherical waves $f_{n, l, m}(r, \Theta, \varphi)=$ $h_{l}^{(1)}\left(k_{n} r\right) Y_{l}^{m}(\Theta, \varphi)$. This calculation was already carried out by one of the authors [10].

Then, according to (7), the total wave function can be written as the sum of an incoming wave $\Psi_{\text {inc }}$ and a scattered wave $\Psi_{\text {scatt }}$ in the laboratory frame. The incident Gordon-Volkov plane wave has then the following form:

$$
\begin{aligned}
& \Psi_{i n c}=\sum_{n, l, m} J_{n}\left(k_{0} a \cos \Theta_{0}\right) i^{l}(2 l+1) \frac{(l-m) !}{(l+m) !} j_{l}\left(k_{0} r\right) \\
& P_{l}^{m}\left(\cos \Theta_{0}\right) P_{l}^{m}(\cos \Theta) \exp \left[i m\left(\varphi-\varphi_{0}\right)\right] \exp \left(-i \omega_{n} t\right) .
\end{aligned}
$$

The scattered waves are outgoing spherical GordonVolkov states with energies $\hbar\left(\omega_{0}+n \omega\right)=\hbar \omega_{n}$, they may be written in terms of ordinary spherical outgoing waves

$\Psi_{\text {scatt }}=\sum_{n^{\prime}, l^{\prime}, m^{\prime}} \sum_{n^{\prime \prime}, l^{\prime \prime}} i^{l^{\prime}-l^{\prime \prime}}\left(2 l^{\prime \prime}+1\right) A\left(n^{\prime}, l^{\prime}, m^{\prime}\right) h_{l^{\prime \prime}}^{(1)}\left(k_{n^{\prime}} r\right)$

$P^{m^{\prime}}\left(l^{\prime}, l^{\prime \prime} ; n^{\prime \prime} \mid k_{n^{\prime}} a\right) P_{l^{\prime \prime}}^{m^{\prime}}(\cos \Theta) \exp \left[i m^{\prime} \varphi\right] \exp \left(-i \omega_{n^{\prime}+n^{\prime \prime}} t\right)$.

where the kernel

$$
P^{m}\left(l, l^{\prime} ; s \mid k_{n} a\right) \equiv \frac{1}{2} \frac{\left(l^{\prime}-m\right) !}{\left(l^{\prime}-m\right) !} \int_{-1}^{1} P_{l}^{m}(x) P_{l^{\prime}}^{m}(x) J_{s}\left(-k_{n} a x\right) .
$$

will be evaluated in the next subsection.

Taking into account (14) and (15) we obtain from the boundary condition (6) in the form

$$
\left[\Psi(r, \Theta, \varphi, t)=\Psi_{i n c}+\Psi_{\text {scatt }}\right]_{r=R}=0
$$

the following matching equations

$$
\begin{aligned}
& J_{n}\left(k_{0} a \cos \Theta_{0}\right) i^{l} \frac{(l-m) !}{(l+m) !} j_{l}\left(k_{0} R\right) P_{l}^{m}\left(\cos \Theta_{0}\right) \exp \left(-i m \varphi_{0}\right) \\
& +\sum_{n^{\prime}, l^{\prime}} i^{l^{\prime}-l} A\left(n^{\prime}, l^{\prime}, m\right) h_{l}^{(1)}\left(k_{n^{\prime}} R\right) P^{m}\left(l^{\prime}, l ; n-n^{\prime} \mid k_{n^{\prime}} a\right)=0
\end{aligned}
$$

where the orthogonality of the spherical harmonics $Y_{l}^{m}(\Theta, \varphi)$ and the orthogonality of the different Fourier components in $t$ have been used. From (18) the so far unknown coefficients $A(n, l, m)$ can be evaluated.

\subsection{Evaluation of the kernel $P^{m}\left(l^{\prime}, l ; n-n^{\prime} \mid k_{n^{\prime}} a\right)$}

The kernels $P^{m}\left(l^{\prime}, l ; n-n^{\prime} \mid k_{n^{\prime}} a\right)$ defined by (16) can be evaluated numerically, but this is a rather demanding task, because high precision is needed, due to the structure of the matching equations. Instead, we outline here, how to compute these quantities accurately and effectively, in terms of of hypergeometric functions, without numerical integration.

We can expand the product of two associated Legendre polynomials in the integrand as a sum of associated Legendre polynomials $[15,16,17]$. We use Balmino's product-sum relationship for the associated Legendre polynomials [15]:

$$
P_{l}^{m}(x) P_{j}^{q}(x)=\sum_{\substack{k=\max (|m+q|,|-j|) \\[l+k+j: \text { even }]}}^{l+j} Q_{l m j q}^{k} P_{k}^{|m+q|}(x),
$$


where

$$
\begin{aligned}
& Q_{l m j q}^{k}=\frac{2 k+1}{2^{l+j+k+1}} \frac{[k-(m+q)] !}{(k+m+q) !} \sum_{p=0}^{m+q}(-1)^{p}\left(\begin{array}{c}
m+q \\
p
\end{array}\right) \\
& \sum_{r=0}^{[(l-m) / 2]}(-1)^{r} H_{l m}^{r} \sum_{s=0}^{[(j-q) / 2]}(-1)^{s} H_{j q}^{s} \sum_{v=0}^{[(k-(m+q)) / 2]}(-1)^{v} H_{k, m+q}^{v} \\
& \frac{1+(-1)^{l+j+k}}{l+k+j+1-2(m+q)+2(p-r-s-v)},
\end{aligned}
$$

with

$$
H_{\alpha \beta}^{\gamma}=\frac{(2 \alpha-2 \gamma) !}{\gamma !(\alpha-\gamma) !(\alpha-\beta-2 \gamma) !} .
$$

We note that for $m=0$ and $p=0$, the expansion is well known, see formula (8.915.5) of Gradshteyn and Ryzhik.[18]

It is now sufficient to determine the integral of the product of one associated Legendre polynomial and a Bessel function of the first kind. The associated Legendre polynomial can be expressed with the ordinary Legendre polynomial

$$
P_{l}^{m}(x)=(-1)^{m}\left(1-x^{2}\right)^{m / 2} \frac{d^{m}}{d x^{m}} P_{l}(x)
$$

Using the power series expansion of the Legendre polynomial

$$
P_{l}(x)=2^{l} \sum_{k=0}^{\infty}\left(\begin{array}{l}
l \\
k
\end{array}\right)\left(\begin{array}{c}
\frac{l+k-1}{2} \\
l
\end{array}\right) x^{k},
$$

we can now write the associated Legendre polynomial in the form

$$
P_{l}^{m}(x)=(-1)^{m}\left(1-x^{2}\right)^{m / 2} \sum_{k=0}^{l}\left(\begin{array}{l}
l \\
k
\end{array}\right)\left(\begin{array}{c}
\frac{l+k-1}{2} \\
l
\end{array}\right) \frac{2^{l} k ! x^{k-m}}{(k-m) !}
$$

The integral of a product of a power function and a Bessel function of the first kind reads

$$
\begin{aligned}
& \int_{-1}^{1} x^{k} J_{s}\left(-k_{n} a x\right)=\frac{2^{l-s-1} \Gamma\left(\frac{m}{2}+1\right)\left(\frac{k+l-1}{2}\right) !}{\left(\frac{k-l-1}{2}\right) !(l-k) !(k-m) !} \\
& \cdot\left[(-1)^{k}\left(k_{n} a\right)^{s}+(-1)^{m}\left(-k_{n} a\right)^{s}\right] \Gamma\left(\frac{k-m+s+1}{2}\right) \\
& \cdot \tilde{F}_{2}\left[\frac{k-m+s+1}{2} ; \frac{k+s+3}{2}, s+1 ;-\frac{\left(k_{n} a\right)^{2}}{4}\right],
\end{aligned}
$$

where ${ }_{1} \tilde{F}_{2}$ is the regularized hypergeometric function defined by

$$
{ }_{1} \tilde{F}_{2}=\frac{{ }_{1} F_{2}\left(a_{1} ; b_{1}, b_{2}, z\right)}{\Gamma\left(b_{1}\right) \Gamma\left(b_{2}\right)},
$$

and ${ }_{1} F_{2}\left(a_{1} ; b_{1}, b_{2}, z\right)$ is a generalized hypergeometric function. Thus the kernels $P^{m}\left(l^{\prime}, l ; n-n^{\prime} \mid k_{n^{\prime}} a\right)$ can be computed with a finite sum of hypergeometric functions. We note that this quantity also appears in recent works on laser-assisted electron scattering, see e.g., Refs. [11, 12].

\section{Results}

We consider the low intensity limiting case where the intensity parameter $\mu \ll 1$. It can be easily shown that the coefficients $A(n, l, m)$ are no longer coupled in that case, and we have an explicit solution for them as

$$
\begin{aligned}
& A(n, l, m)=-i^{l}(2 l+1) \frac{(l-m) !}{(l+m) !} \frac{j_{l}\left(k_{0} R\right)}{h_{l}^{(1)}\left(k_{n} R\right)} \\
& \cdot P_{l}^{m}\left(\cos \Theta_{0}\right) \exp \left(-i m \varphi_{0}\right) J_{n}\left(k_{0} a \cos \Theta_{0}\right) .
\end{aligned}
$$

The total differential cross sections can be obtained through the asymptotic form of the scattered wave function. Inserting (27) into the expression of the scattered wave function and taking the limit $r \rightarrow \infty$, the total differential cross sections read

$$
\begin{aligned}
\frac{d \sigma_{n}}{d \Omega}= & \frac{k_{n}}{k_{0}}\left|\frac{1}{k_{n}} \sum_{l=0}^{\infty}(2 l+1) \frac{j_{l}\left(k_{0} R\right)}{h_{l}^{(1)}\left(k_{n} R\right)} P_{l}\left(\cos \gamma_{0}\right)\right|^{2} \\
& \cdot J_{n}^{2}\left(k_{0} a \cos \Theta_{0}\right),
\end{aligned}
$$

where

$$
\cos \gamma_{0}=\cos \Theta \cos \Theta_{0}+\sin \Theta \sin \Theta_{0} \cos \left(\varphi-\varphi_{0}\right)
$$

The wave function (5) is expressed in terms of a nested infinite series. It is obvious that an analytic expression cannot be achieved, numerical evaluations are required. The accuracy of the wavefunction and the total differential cross sections depends on the truncation of the infinite series. In our results, the boundary condition (17) is accurately satisfied, i.e., the real and the imaginary part of the wave function on the boundary is less than $10^{-6}$, if we choose the upper limit of the series to be $L=50-60$, depending on various parameters.

First, we show plots of the logarithm of the probability density of the total wave function (10), in the $x-z$ plane, around a hard sphere of $5 \mathrm{~nm}$ radius, without the laser field in Figure 2, and in the presence of the laser field in Figure 3 (assuming the weak field limit). In the presence of the laser field, interference fringes appear in the shadow region behind the nano-sphere, due to the multiphoton processes between the laser field and the electron. 
Figure 4 shows the total differential cross section of the $n$th scattering channel as a function of the polar angle $\Theta$, in a polar plot. Since we choose $\Theta_{0}=0$ for the incoming electrons, it is plausible to study the directional dependence in the $x-z$ plane where the azimuth $\varphi$ is zero. These plots show that the more energy is lost or gained by the electrons in form of photons, the wider (and in the case of energy loss also the more structured) the angular dependence becomes, along with increasing possibility of backscattering.

In Figure 5, we focus on forward scattering, where the scattering angle $\Theta$ is chosen to be zero and we set the values for the field strength such that the weak field limit condition still holds. The total differential cross section is plotted as a function of the electron energy in the $n$th channel. The positive and the negative values of $n$ correspond to stimulated photon absorption and emission, respectively. It is clearly shown by this plot, that the sidebands $n \neq 0$ get more populated with increasing laser field strength [9].

If the low intensity assumption is released, then the linear system of equations is coupled, and we can truncate the system only at a much larger value of $n$, in order to ensure convergence. At a qualitative level, we expect that the higher order sidebands get more populated at the expense of the central ( $n=0)$ band's significant reduction, and that the interference patterns in the electron probability density become more pronounced.

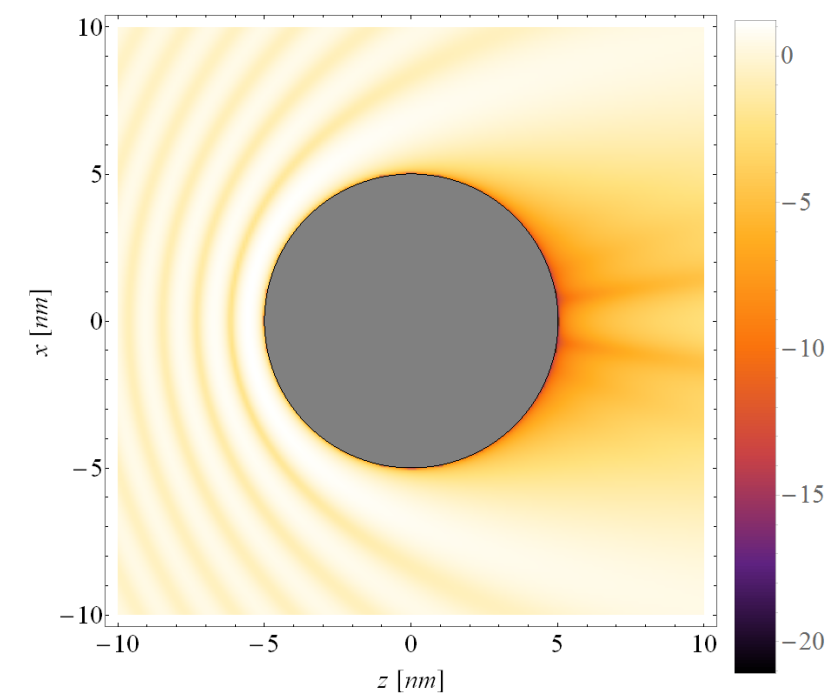

Figure 2: Density plot of the logarithm of the probability density of the total electron wave function, in the $x-z$ plane, around a hard sphere of $5 \mathrm{~nm}$ radius, in the absence of the laser field, with incoming electron energy $E_{0}=0.25 \mathrm{eV}$.

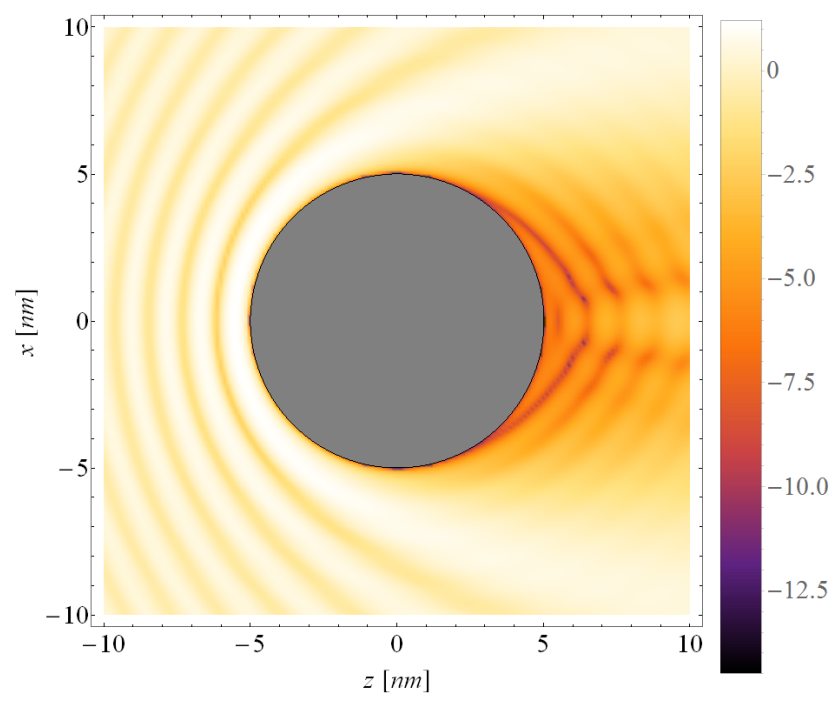

Figure 3: Density plot of the logarithm of the probability density of the total electron wave function, in the $x-z$ plane, around a hard sphere of $5 \mathrm{~nm}$ radius, assuming the weak field limit. Parameters: incoming electron energy $E_{0}=0.25 \mathrm{eV}$, photon energy $\hbar \omega=1.5 \mathrm{eV}$.

\section{Summary}

We presented theoretical results in connection with laser-assisted electron scattering on a nano-sphere. We outlined a novel method for the accurate and effective computation of the kernel necessary for the solution of the matching equations. We presented and analysed particular results regarding the total differential scattering cross sections. Our results can also be applied for characterizing the multiphoton-multipole components of the electron de Broglie waves scattered by nanostructures like metal nanoparticles embedded in dielectrics.

\section{Acknowledgment}

S. Varró has been supported by the National Scientific Research Foundation OTKA, Grant No. K 104260. The project was partially funded by "TÁMOP-4.2.2.D15/1/KONV-2015-0024ELITeam' - Establishment of the ELI Institute at the University of Szeged: foundation of interdisciplinary research in the field of lasers and their applications", which is supported by the European Union and co-financed by the European Social Fund. Partial support by the ELI-ALPS project is also acknowledged. The ELI-ALPS project (GOP-1.1.1-12/B2012-0001) is supported by the European Union and cofinanced by the European Regional Development Fund. 


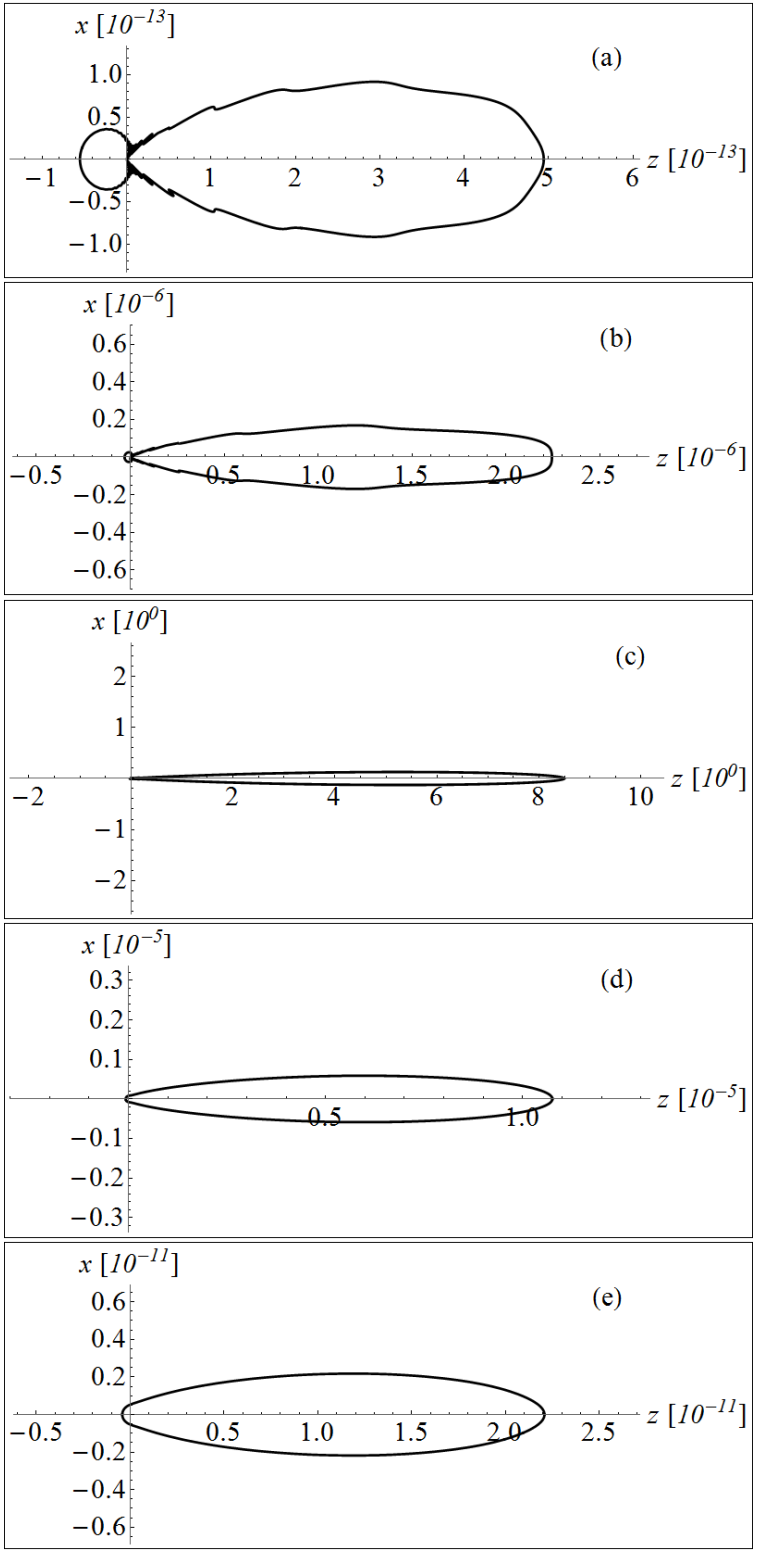

Figure 4: Polar plots of the total differential cross section as a function of the polar angle, in relative units normalized to the maximum of the $n=0$ case, for (a) $n=-2$, (b) $n=-1$, (c) $n=0$, (d) $n=1$, (e) $n=2$. Parameters: incoming electron energy $E_{0}=4 \mathrm{eV}$, photon energy $\hbar \omega=1.5 \mathrm{eV}$, field strength $2.5711 \times 10^{7} \mathrm{~V} / \mathrm{m}$.

\section{References}

[1] M. V. Fedorov, Atomic and Free Eletrons in a Strong Laser Field, World Scientific, 1997.

[2] T. Brabec, F. Krausz, Intense few-cycle laser fields: Frontiers of nonlinear optics, Rev. Mod. Phys. 72 (2000) 545-591.

[3] P. B. Corkum, F. Krausz, Attosecond science, Nat. Phys. 3 (6) (2007) 381-387.

[4] S. Varró, G. Farkas, Attosecond electron pulses from interfer-

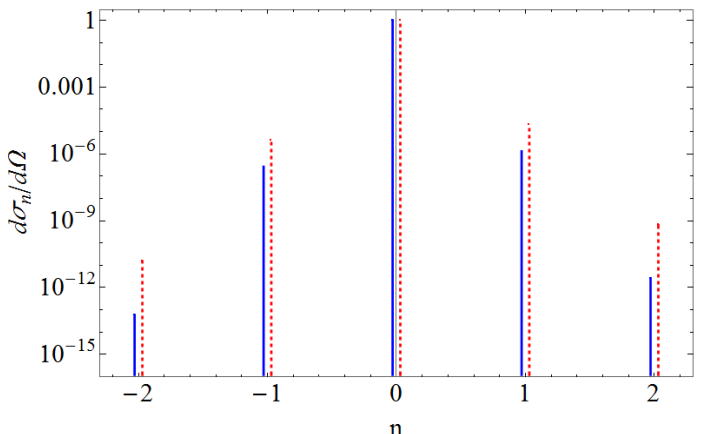

Figure 5: Total differential cross section at the forward direction $(\Theta=$ 0 ) as a function of the scattered electron energy $E_{n}$. The red dashed and the solid blue lines correspond to field strengths $1.0284 \times 10^{8}$ and $2.5711 \times 10^{7} \mathrm{~V} / \mathrm{m}$ respectively, the incident electron energy is $4 \mathrm{eV}$, photon energy $\hbar \omega=1.5 \mathrm{eV}$.

ence of above-threshold de broglie waves, Laser and Particle Beams 26 (2008) 9-20.

[5] W. E. King, G. H. Campbell, A. Frank, B. Reed, J. F. Schmerge, B. J. Siwick, B. C. Stuart, P. M. Weber, Ultrafast electron microscopy in materials science, biology, and chemistry, Journal of Applied Physics 97 (2005) 111101

[6] A. H. Zewail, Four-dimensional electron microscopy, Science 328 (5975) (2010) 187-193.

[7] S. T. Park, A. H. Zewail, Enhancing image contrast and slicing electron pulses in $4 \mathrm{~d}$ near field electron microscopy, Chemical Physics Letters 521 (0) (2012) 1 - 6.

[8] M. L. Sang T. Park, A. H. Zewail, Photon-induced near-field electron microscopy (pinem): theoretical and experimental, New J. Phys. 12 (12) (2010) 123028.

[9] B. Barwick, D. J. Flannigan, A. H. Zewail, Photon-induced near-field electron microscopy, Nature 462 (7275) (2009) 902906.

[10] S. Varró, F. Ehlotzky, Scattering by a hard sphere in a laser field, Zeitschrift fr Physik D Atoms, Molecules and Clusters 8 (3) (1988) 211-217.

[11] N. Morrison, C. H. Greene, Laser-assisted electron-argon scattering at small angles, Phys. Rev. A 86 (2012) 053422.

[12] L. W. Garland, A. Jaron, J. Z. Kaminski, R. M. Potvliege, Off-shell effects in laser-assisted electron scattering at low frequency, Journal of Physics B: Atomic, Molecular and Optical Physics 35 (13) (2002) 2861.

[13] D. M. Volkov, Z. Phys. 94 (1935) 250.

[14] W. Gordon, Z. Phys. 40 (1926) 117.

[15] G. Balmino, M. Sidlichovsky, On the product of legender functions as encountered in geodynamics, Studia Geophysica et Geodaetica 22 (2) (1978) 107-118.

[16] G. Giacaglia, M. Bursa, Transformations of spherical harmonics and applications to geodesy and satellite theory, Studia Geophysica et Geodaetica 24 (1) (1980) 1-11.

[17] C. Hwang, A method for computing the coefficients in the product-sum formula of associated legendre functions, Journal of Geodesy 70 (1-2) (1995) 110-116.

[18] I. S. Gradshteyn, I. M. Ryzhik, Table of Integrals, Series and Products, 7th Edition, Academic Press Inc., 2007. 